\title{
Discussions philosophiques avec des adolescents empêchés de penser. Didactique clinique, résilience et théories de la complexité
}

Philosophical discussions with adolescents prevented from thinking. Clinical didactics, resiliency and theories of complexity

Johanna Henrion-Latché

\section{OpenEdition \\ Journals}

Édition électronique

URL : http://journals.openedition.org/trema/6137

DOI : $10.4000 /$ trema.6137

ISSN : 2107-0997

Éditeur

Faculté d'Éducation de l'université de Montpellier

Référence électronique

Johanna Henrion-Latché, « Discussions philosophiques avec des adolescents empêchés de penser

Didactique clinique, résilience et théories de la complexité », Tréma [En ligne], 54 | 2020, mis en ligne le 01 décembre 2020, consulté le 14 décembre 2020. URL : http://journals.openedition.org/trema/6137 DOI : https://doi.org/10.4000/trema.6137

Ce document a été généré automatiquement le 14 décembre 2020.

Trema 


\title{
Discussions philosophiques avec des adolescents empêchés de penser. Didactique clinique, résilience et théories de la complexité
}

\author{
Philosophical discussions with adolescents prevented from thinking. Clinical \\ didactics, resiliency and theories of complexity
}

Johanna Henrion-Latché

\section{Introduction}

1 Les théories de la complexité décrites depuis plus de vingt années par Edgar Morin sont au cœur des recherches en éducation (Cyrulnik \& Morin, 2010). La science progressant au rythme de l'évolution d'une société complexifiée, les travaux sur l'Intelligence Artificielle décrivent un type d'enseignement dans lequel les laissés pour compte de la programmatique seraient astreints à recevoir une éducation critique leur permettant de comprendre et s'adapter aux technologies de demain (Alexander, 2017). Au-delà des idéologies et des projections futuristes, d'aucuns font appel à la pensée comme un processus inégalable (Gabriel, 2019) pour apprendre à s'orienter de manière éthique et critique par le biais de l'éducation et des apprentissages (Taddei, 2018 ; Dehaene, 2018) dans les mutations sociétales engendrant la complexité. Or la formation à l'esprit critique, qui mobilise les notions de savoirs discursifs et d'intelligence visant la conceptualisation pour la prise de décision, se heurte au paradigme de la complexité d'adolescents dits "empêchés de penser» (Boimare, 2008), freinant dans certains établissements sensibles, la mise en activité du pensé collectif. Deux conceptions du savoir et de l'intelligence se polarisent alors dans nos sociétés (entre le savoir révélé par l'institution et celui qui consiste à mobiliser l'intelligence par la capacité de discussion, du questionnement, de l'argumentation et du doute), qui peinent à voir émerger des pratiques innovantes telles les discussions à visée philosophique, pourtant 
implémentées dans de nombreux pays depuis plus de cinquante années maintenant (Lipman, Sharp \& Oscanyan, 1980). Au lycée, expérimentalement, ces discussions étayées de supports ad hoc mettent en jeu des savoirs réflexifs et des concepts situés autour de situations discutées en groupe. Si ces supports traitent du principe de résilience, il est possible de mettre en œuvre au cœur des apprentissages une didactique en appui sur les principes d'une didactique clinique (Frisch, 2016, 2018 et à paraître) qui se révèle, face aux nouveaux défis de l'école et des sociétés, nécessaire dans les métiers de l'humain. Selon les préconisations d'Edgar Morin qui visent à renforcer l'humain afin qu'il possède une bonne connaissance de l'environnement dans lequel il vit, donc de la culture qui l'imprègne, les discussions philosophiques avec des adolescents hébétés, empêchés de penser, constituent un moyen de penser la complexité qui "est le défi à affronter" (Morin, 2005). Nous proposons dans cette contribution d'illustrer à l'aide de corpus ${ }^{1}$ en quoi les séances de discussion à visée philosophique à l'école peuvent être un pilier pour la formation à l'éducation complexe (Sasseville et Gagnon, 2007 ; Sasseville, 2019) à l'aide de la didactique clinique, issue des travaux de l'équipe IDEKI ${ }^{2}$ (didactique et métiers de l'humain). Les corpus choisis sont ceux d'une classe d'adolescents de CAP des métiers du bâtiment (6 garçons/1 fille), d'un âge moyen de 17 ans, porteurs de troubles des apprentissages, de la parole et du comportement. Les extraits illustrent la complexité des pensées d'adolescents porteurs de troubles du langage et peu familiers avec la prise de parole ou l'activité argumentative (Boimare, 2008), révélant la vulnérabilité (Jellab et Marsollier, 2018) des établissements en prise avec une nouvelle clinique de la mondialité (Dérivois, 2010). Les séances étayées d'un support écrit ad hoc (Henrion, 2012) questionnaient le principe de résilience injecté dans des savoirs anthropologiquement et scolairement situés (Henrion-Latché, 2016), et examinaient le déploiement des compétences discursives des adolescents visant le discours résilient. Le présent article vise à présent à étudier l'impact de la didactique clinique en DVP par la catégorisation des discours adolescents, selon les trois conceptions épistémologique, praxéologique et psychologique de la didactique clinique.

\section{Philosopher à l'école : didactique du philosopher et didactique clinique}

2 La discussion philosophique ou discussion à visée philosophique (DVP) puise ses racines dans la PPE (philosophie pour enfants) mise au point par Mathew Lipman à l'université de Saint Clair aux États-Unis dans les années 70. Philosophe pragmatiste, professeur confronté aux difficultés de ses étudiants à penser, il mit au point une méthode pédagogique novatrice destinée à être appliquée dès les petites classes, permettant de philosopher avec des enfants et des adolescents. Face au constat de classes hétérogènes d'enfants venus de confluents différents, il misa sur le développement d'habiletés (du langage, de la pensée) par la mise en dialogue philosophique de situations extraites d'historiettes anthropologiquement situées. Dans les pas des méthodes actives de John Dewey (didactique), en appui sur le développement de l'enfant (psychologique) et dans la tradition de la réflexion philosophique occidentale (logique, logos, cogito, etc.), il a construit avec l'aide de son assistante Ann Margaret Sharp, une méthodologie complète composée d'historiettes et de livrets explicatifs destinés aux enseignants désirant mettre en œuvre des discussions dans les classes. Il envisageait de développer la culture 
de la question, du raisonnement, par l'activité dialogique/discursive autour de l'élaboration de différentes habiletés de pensée (Lipman, 1995). Il visait également le déploiement $\mathrm{du}$ bien pensé qui est, pour Edgar Morin «le mode de penser qui permet d'appréhender ensemble le texte et le contexte, l'être et son environnement [...] qui nous permet de comprendre les conditions objectives et subjectives $d u$ comportement humain» (Morin, 2005, p.122). Par l'organisation d'une classe sur le mode d'une communauté de recherche philosophique (CRP), il appliqua les principes d'une parole démocratiquement partagée et une exigence critique par le devoir d'argumentation et du droit d'expression, analysés sous les échanges de cognitions.

3 La philosophie, discipline, y est vécue sur le mode du dialogique (dia-logos) socratique, qui rejette les dogmes, les certitudes, les croyances, et cultive le doute et le questionnement partagé. Ce type de dialogue (pragmatique) «met un accent particulier sur la reconstruction de l'expérience individuelle et sociale et [...] comprend l'éducation comme un processus réflexif toujours en mouvement» (Daniel, 2012, p. 161). Le dialogue philosophique s'y définit par une "interaction sociale rapprochée [...] ouverte par une question déterminée qui fait problème par ses enjeux et sa difficulté, qu'un groupe animé est appelé à examiner en vue d'y chercher des réponses" (Tozzi, Meirieu \& Huguet, 2002). Étudié au sens fort (Daniel \& Delsol, 2010; Auriac-Slusarczyk, 2010), il ouvre la boucle conversationnelle (Trognon, 1991, 1995) qui n'est jamais bouclée, puisque relancée par l'activité de l'animateur. Il s'agit moins d'un mode d'échange spontané qu'un processus qui requiert apprentissage et modalité d'investissement du langage et de la transmission culturelle (Dehaene, 2018, p. 239).

4 En France, ces discussions sont implantées dans les petites écoles depuis 2015, contrairement au lycée professionnel où la philosophie est même absente du programme de terminale. Elles permettent entre autres aux enfants de comprendre « comment à acquérir une sorte de conscience de la vie, un art de prendre du recul par rapport aux choses et aux émotions » (Blanquer \& Morin, 2020, p.27).

\section{La didactique du philosopher}

5 Les discussions philosophiques et la philosophie en particulier font l'objet d'analyses didactiques étudiées depuis plus de vingt ans par le philosophe Michel Tozzi. Par didactique, nous entendons la «science qui étudie pour un domaine particulier, les phénomènes d'enseignement, les conditions de la transmission de la culture propre à une institution et les conditions de l'acquisition des connaissances par un apprenant " (Joshua \& Dupin, 1989) qui «s'appuie sur l'analyse des conduites et des discours produits par les sujets en formation, sur l'analyse des pratiques, des choix et des décisions des enseignants ou autres formateurs [...]» (Astolfi \& Delevay, 1989, p. 21). Concernant les DVP, nous parlons de la didactique du philosopher.

La matrice didactique de Michel Tozzi PAC (problématiser-argumenter-conceptualiser) est une méthode permettant aux enseignants, animateurs, non-philosophes, de mettre en œuvre des discussions philosophiques dans une exigence du philosopher (Tozzi, 2009 ; Henrion-Latché \& Tozzi, 2016; Henrion-Latché, 2019). Elle permet d'enclencher des discussions faisant appel à la complexité philosophique dans laquelle le sens des mots nait de la régulation interlocutoire. Exercées en groupe sous la guidance d'un animateur, elles travaillent la régulation des membres par un système de signes partagés permettant la mise en rituelle et en norme d'un espace d'interactions 
démocratiques. Une régulation de la communauté interlocutoire s'opère de manière interne sans que des parasitages externes autres que le langage et l'activité du pensé viennent perturber les raisonnements échangés. C'est principalement l'action didactique disciplinaire du professeur qui est pointée $($ Go, 2007) qui détermine à la fois les formes de communication produites, les énoncés philosophiques distribués et le référencement proprement philosophique qui se construit sous l'action du professeur.

La matrice didactique peut être étayée de différents supports en fonction de l'objectif défini par l'animateur et par les membres du groupe (littérature classique, de jeunesse, contes, mythes, bande dessinée, supports ad hoc, etc.). Le rôle du maître s'y définit sous une déontologie précise, garantissant la loi dans les groupes, tout en préservant la construction de liens afin que les adolescents puisent construire le sens des questions existentielles (la vie, la mort, le désir, etc.) ou pour reprendre les termes de Morin, « les problèmes fondamentaux et les problèmes globaux [qui ont été] évacués des sciences disciplinaires [et ne] sont sauvegardés que dans la philosophie, mais cessent d'être nourris des apports de la science » (Morin, 2000, p. 46)

8 Cependant, avec des adolescents dysfonctionnels, la matrice seule, bien que nécessaire, n'est pas suffisante. Pour exemple, les élèves dits à besoins éducatifs particuliers ont besoin d'être "portés psychiquement par un enseignant, dans la qualité de leur relation quotidienne». Et de se questionner sur «l'accent [...] mis sur les potentialités affectives, cognitives, et créatrices de l'élève ou sur ses faiblesses » (Dérivois, 2010, p. 68). Ainsi, la didactique du philosopher nécessite également de s'intéresser aux impacts de la discussion du côté des élèves sous l'angle des acquis psychiques (Lévine \& Moll, 2004) qui focalise principalement sur les apports (couts, bénéfices, pertes) de l'enfant faisant l'expérience de son propre cogito. Jacques Lévine nous instruit que la motivation des élèves à philosopher se rapporte à trois sortes de pulsion : «la pulsion d'équivalence, la pulsion de découverte et la pulsion de civilisation». La première renvoie l'élève à l'interlocuteur valable, la seconde renvoie au plaisir de découvrir le monde, le « vouloir savoir en philosophie» et la troisième est celle faire en sorte que les sujets soient confrontés à des forces contraires afin qu'ils aient le désir d'être des représentants de la civilisation (Lévine \& Moll, 2004, p.103). Il s'agit de mobiliser l'activité pulsionnelle d'un élève dans une culture ouverte qui permet des dialogues et des échanges d'idées, tout en faisant de l'école le «lieu d'apprentissage des savoirs, mais aussi lieu d'élaboration d'un certain rapport au savoir sur l'autre, sur soi » (Dérivois, 2010, p. 69). Si le pulsionnel de l'élève est convoqué, il importe d'étudier l'impact de ce pulsionnel dans le rapport aux représentations enseignantes et dans les réactions (positives ou négatives) de ce pulsionnel sur l'adulte. La didactique clinique est alors requise et complète la didactique du philosopher.

9 Afin de contrer tout dérapage ou toute fausse interprétation des apports de la didactique clinique en analyse des discussions philosophiques, il importe de recentrer l'analyse des activités par les résultats des interactions et des interlocutions de groupe. Par les apports de l'histoire et de l'épistémologie de la discipline, la visée socratique et maïeutique puise au fondement de la pratique les conditions favorables à l'émergence d'une nouvelle didactique clinique. Appliquée aux cadres discursifs dans une nouvelle complexité, l'enseignant/animateur vecteur et transmetteur d'un enseignement extrait de son rapport à son propre savoir. Il recentre l'activité de transmission de l'humain à l'humain, renversant les apports des «machines» qui n'enseigneront «jamais rien 
puisqu'il faut s'enseigner à soi-même pour transmettre » (Coutel, 2000). La pensée est du coté des élèves, et du coté du maître qui questionne.

\section{Du philosopher vers une didactique clinique}

10 La didactique (ici clinique) est définie comme un " art d'enseigner » qui se différencie de la «zététique» (qui est l'art de rechercher) (Coutel, 2000, p.48). En ce sens, la didactique s'intéresse autant à l'enseignant comme initiateur et transmetteur de son enseignement qu'aux contenus transmis. Par la didactique clinique, nous ouvrons un nouveau champ d'intérêt dans les DVP en focalisant sur les choix de l'enseignant, sur sa posture, et sur l'enseignement qu'il souhaite transmettre au sein des discussions philosophiques. La philosophie faisant appel à la pensée complexe, elle porte une responsabilité éthique et professionnelle envers les adolescents. Et s'il est visé un rebond de la pensée, une attention déontologique et clinique particulière doit être posée sur l'activité du maître (Henrion-Latché \& Tozzi, 2016) par le recours à la didactique.

«La didactique étudie les conditions de transmission et de construction des enseignements et des apprentissages dans des disciplines, des éducations, des domaines émergents, des domaines professionnels [...] la didactique clinique étudie la transmission des savoirs à travers le sujet enseignant qui est considéré comme un sujet unique, empreint de son histoire personnelle » (Frisch, 2016, 2018).

11 L'intérêt d'élaborer une didactique clinique au service du philosopher prend appui sur le processus de didactisation des travaux de Frisch \& Paragot (2015), selon les trois orientations décrites par Philippe Jonnaert et Suzanne Laurin (2001) : épistémologique (si la réflexion porte essentiellement sur les objets d'enseignement et d'apprentissage); praxéologique (si la réflexion porte essentiellement sur l'intervention didactique dans les classes); et psychologique (si la réflexion porte essentiellement sur le sujet qui apprend) (Frisch, 2007 , p. 158). Pour ce faire, nous nous sommes dotés d'outils d'analyse du discours et d'analyse de contenus (Henrion-Latché \& Auriac-Slusarczyk, 2020) et d'extraits de corpus issus d'expérimentations consultables sur le site " philosophèmes ». À l'aide d'« analyseurs » (Frisch, 2008), la didactique clinique s'appuie sur des fondements scientifiques afin de rendre compte de la portée d'un enseignement sur un sujet inscrit dans un éco système (social, familial, scolaire) en vue d'être intégré dans un méta-système (Morin, 2005, p. 65).

Dans les DVP, le savoir mis en jeu dans les discussions par le biais de supports écrits ad hoc est discuté à partir de leurs connaissances, de leur expérience et de leurs représentations antérieures sur le monde. La didactique clinique est ici convoquée comme " processus de restructuration des acquis antérieurs, processus sur lequel il y a peutêtre possibilité d'intervention » (Frisch 2008, p.7). À partir des échanges des adolescents de groupe s'installe une relation de sujets à sujets dans laquelle l'adolescent se vit comme sujet pensant, validant le postulat que "la subjectivité n'a pas de sens en dehors de l'intersubjectivité» (Vavassori \& Harrati, 2018, p.17). Cette intersubjectivité est analysée sous l'approche des liens construits collectivement par le biais du savoir sous l'impulsion de l'animateur/enseignant, qui utilise son « méta point de vue ». Ce dernier "n'est possible que si l'observateur concepteur s'intègre dans l'observation et dans la conception. Voilà pourquoi la pensée de la complexité a besoin de l'intégration de l'observateur et $d u$ concepteur dans son observation et sa conception » (Morin, 2005 p.102). Par la conception de séances porteuses, la possibilité de faire émerger le sujet, de se détacher des 
anciennes emprises invalidantes (mutisme, réclusion scolaire, échec et répétition victime/délinquance) implique de concevoir l'adolescent comme un être à la fois suffisant et à la fois insuffisant à lui-même (Morin, 2005, p.53). En construisant des liens, en incorporant la loi dans les DVP, tout en élaborant collectivement le sens par la pensée philosophique, il est possible, entre élèves et enseignants, de "remplacer une pensée qui sépare et qui réduit par une pensée qui distingue et qui relie 》(Morin, 2000, p.53).

\section{La complexité au cœur de l'éducation}

La complexité qui s'invite dans nos sociétés interroge les chercheurs en éducation en proie avec des problématiques en établissements scolaires dits "sensibles", matérialisées dans les défauts du pensé des élèves. Échec scolaire et/ou social, vulnérabilités et inégalités socio-économiques sont des marqueurs rencontrés pour partie dans certains lycées professionnels, concentrant dans certaines spécialités des élèves issus de l'éducation prioritaire ou spécialisée, des adolescents concernés par les champs du handicap et de l'inclusion autrement nommés "à besoins éducatifs particuliers » (Henrion-Latché \& Auriac-Slusarczyk, 2020). Passages à l'acte, défiance envers l'adulte, défauts du langage, de la parole, de la pensée, absentéisme, décrochage et vulnérabilité sociale et scolaire (Jellab et Marsollier, 2018) sont autant d'indicateurs renvoyant aux défauts de l'abstraction, de la réflexion, de la subjectivation et de la pensée. Ce constat s'illustre pour exemple dans les derniers résultats des tests PISA 2019, qui révèlent que moins d'un élève sur dix (élèves de quinze ans testés dans les 79 pays de l'OCDE) est aujourd'hui incapable de distinguer un fait d'une opinion. De nombreux chercheurs sur la clinique de l'adolescence (Troadec, 2007 ; Dérivois, 2010, Moro, 2017) exposent depuis ces dix dernières années des constats similaires invitant à contextualiser les mutations sociales, sociétales, économiques et politiques d'une mondialisation qui dérégule la connaissance et la pensée, et nécessite de prendre en compte une clinique de nouveaux symptômes contemporains (Vavassori \& Harrati, 2018). En effet, "plus les problèmes deviennent planétaires, plus ils deviennent impensés. Incapable d'envisager le contexte et le complexe planétaire, l'intelligence aveugle rend inconscient et irresponsable " (Morin, 2005, p. 49). La question de la pathologie de la pensée (Morin, 2005, p.23) reste comme en suspens, dans une école en mutation (digitale, numérique) dans laquelle la surabondance de l'information nécessiterait de recourir à l'intelligence (Alexandre, 2017, p. 104). Par intelligence, les chercheurs sur l'intelligence artificielle ${ }^{3}$ entendent des capacités : "de discrimination, de synthèse et d'articulation créative " (Alexandre, op.cit). Or, transmettre des capacités par le biais de différents savoirs pour parvenir à évaluer des compétences nécessite de recourir à l'activité discursive et conversationnelle avec l'élève (Henrion-Latché \& Frisch, 2019). Cette approche amorce une appréhension de la complexité dans les cadres de l'éducation.

\section{Complexité et éducation}

14 La complexité décrite par Edgar Morin se définit comme "un tissu (complexus : qui est tissé ensemble) de constituants hétérogènes inséparablement associés" selon un «tissu d'événements, actions, interactions, rétroactions, déterminations, aléas, qui constituent notre monde phénoménal» (Morin, 2005, p.21). Ce «tissu interdépendant, interactif et inter 
rétroactif entre l'objet de connaissance et son contexte» introduit "la complexité [qui] est, de ce fait, le lien entre l'unité et la multiplicité» (Morin, 2005 p.43).

Appliquée aux cadres des apprentissages, la complexité peut être une réponse permettant de poser des pratiques novatrices propices à la création de liens par le biais de l'intelligence collective (intelligence, de intellegentia, qui est la faculté de comprendre, dont le préfixe inter signifie « entre » et dont le radical ligare signifie " lier»). L'intelligence conçue par les chercheurs en didactique clinique (Frisch \& Paragot, 2015) est ce qui permet de relier des éléments qui, sans elle, resteraient séparés. C'est l'idée de la mutualisation des savoirs, du partage des connaissances et donc, à l'école, de l'interdisciplinarité qui est convoquée. Cette idée déjà au cœur des recherches sur le numérique éducatif par la place que doit donner l'école «aux travaux collaboratifs, et [à l'] entraide entre pairs" (Alexandre, 2017, p.196). La question des méthodes collaboratives et coopératives des élèves par des méthodes actives et discursives est au cœur des recherches en didactique, qui s'intéresse à la place de l'enseignant comme garant de l'intelligence collective mise en jeu dans ces séances. Cependant, l'aspect novateur de la didactique clinique est qu'elle s'intéresse à l'activité de l'enseignant en le conduisant vers l'analyse in fine de sa propre pratique. L'objectif visé est que celui-ci soit à la fois l'acteur et l'observateur de la mise en œuvre du lien de groupe, lien « inséparable entre l'observateur et la chose observée » (Morin, 2005, p. 19).

L'intelligence est ainsi convoquée non plus comme caractéristique ou faculté isolée, mais comme " une intelligence générale nécessitant le libre exercice de la curiosité » que l'adulte doit éveiller (Morin, 2005, p. 44). Simplement, quand les apprentissages adolescents ont été altérés, quand ils "soulèvent autant de vécus destructeurs que de volontés de réparation » alors «(...) Apprendre et penser exposent à toute une série de dangers qui, lorsqu'ils ne peuvent être supportés, conduisent à l'échec, tout autant scolaire que social » (Vavassori et Harrati, 2018, p. 53). La capacité à se « saisir par la pensée, à emporter de soi une parcelle de la réalité, un modèle de la structure du monde » (Dehaene, 2018, p.37) n'est plus assurée. Elle se heurte aux réalités des éducations et des enseignements dans lesquels la place du savoir ne prend plus sens, où la complexité des classes affronte la complexité des curriculums, des apprentissages, eux-mêmes parasités par des problèmes rendant les adolescents indisponibles aux liens insufflés par l'adulte.

17 L'intelligence devient le paradigme sur lequel prend appui l'adulte, l'éducateur, l'enseignant, ne se contentant plus de transmettre un savoir désincarné, mais appelé à faire vivre, durant quelques heures, des savoirs en circulation par l'approche d'une didactique clinique fondée sur la pensée complexe, selon les dix pistes d'activation d'Edgar Morin (2005): «1. Changer de paradigme; 2: relier tous les points de vue; 3. Appliquer le principe d'irréductibilité; 4: utiliser la pensée dialogique; 5 . dépasser la controverse société/individu; 6. appliquer le principe de récursivité; $7:$ ne pas avoir peur du désordre; 8 ; être stratège; 9. toute action est un pari et 10 : l'autocritique est une hygiène permanente ». Ces préconisations sont au cœur des discussions philosophiques, articulant discipline de la philosophie, activité dialogique partagée avec les adolescents et questionnements autour des grandes thématiques humaines et plus encore, quand est visé le rebond résilient (Auriac-Slusarczyk \& Henrion-Latché, 2018). 


\section{Discussions philosophiques et pensée complexe}

18 Si toute éducation vise à "enseigner à vivre " (Blanquer et Morin, 2020, p. 9), il est question de donner à des adolescents empêchés de penser (Boimare, 2008) quelques heures par semaine, des espaces collectifs de réflexion sur les grandes thématiques humaines, à hauteur d'adolescents reconnus comme «auditeurs valables" (Tozzi \& Solère-Queval, 2004, p.80; Tozzi, 2007). Ces séances de DVP, organisées en référence à la didactique clinique, visent des apprentissages ciblés de l'abstraction, de la réflexion, de la subjectivation et de la pensée.

Cette éducation (philosophique) d'une adolescence (dogmatique) prend appui sur sept principes «1. Les cécités de la connaissance (à partager) : l'erreur et l'illusion (des croyances vs la recherche de vérité); 2 . les principes d'une connaissance pertinente (la méthode de recherche et d'investigation); 3. enseigner la condition humaine (par la philosophie); 4. enseigner l'identité terrienne (par les discussions partagées dans l'interculturel des classes); 5. affronter les incertitudes (par l'appui de l'enseignant) ; 6. enseigner la compréhension (par la visée démocratique des discussions); et 7. l'éthique du genre humain (par les valeurs et les normes partagées)»(Morin, 1999, p.55). Ces principes constituent l'essence de la philosophie et de ses méthodes maïeutiques/ socratiques, tels que travaillées avec des adolescents aux défauts du pensé dans les classes (Richaud, 2014). Par les échanges de pensées discutées entre adolescents et l'enseignant/animateur, des pensées articulées conduisent à la pratique de la "pensée complexe » en communauté de recherche (Sasseville, 2019). Les pensées, préalablement à être produites, "sont déjà reçues » (Gabriel, 2019, p.49) et permettent le rebond sur la pensée des autres. La méthode dialogique permet de travailler les consciences collectives en faveur de la conscience individuelle, permettant l'avènement de la pensée au détriment des modèles de pensées. L'épistémologie, entendue comme la connaissance de la connaissance, construite entre élèves permet l'élaboration d'hypothèse, de raisonnement, à partir de l'expérience ou du point de vue des adolescents. La critique s'imbrique dans la complexité en remettant en jeu les certitudes, les injonctions, les pensées toutes prêtes ou déjà pensées en créant une reliance cognitive. L'esprit critique se décalant ainsi de l'esprit de critique, les discussions philosophiques offrent des moments de lecture collective d'un chapitre de livre, une activité de résumé, de discussions et de critique des contenus, dans la promotion d'un « enseignement qui aurait vocation à stimuler l'esprit critique, mais également l'esprit autocritique et la connaissance de soi» (Blanquer et Morin, 2020, p.10) par l'apprentissage du problématiser.

20 Au sein des recherches sur les modalités du pensé mises en œuvre dans les discussions philosophiques, nous devons à Marie France Daniel d'avoir élaboré un modèle du processus de développement d'une pensée critique/dialogique (Daniel, 2007 ; Daniel \& Gagnon, 2011). Le modèle permet de mettre en lumière le développement épistémologique et d'observer l'évolution des échanges philosophiques dans les discussions. Ce processus de développement d'une pensée critique/dialogique (catégorisées dans l'égocentrisme, le post égo, le pré relativisme, le relativisme, le post relativisme, la pré intersubjectivité/intersubjectivité) (Daniel, 2012, p. 5) repose sur le postulat de la "pensée complexe, que d'aucuns appellent critique» (Daniel, 2012). Cette pensée dans les DVP est identifiable à la manière de penser des enfants et des adolescents, à partir des contenus des échanges lors des discussions. La complexité ici 
entendue est celle qui s'articule dans la dialectique par l'apprentissage de la contradiction, de la réversibilité des pensées, du discernement du vrai ou du faux, de la recherche de la vérité, prémisses à toute éducation libérale. Est initialement visé le développement intellectuel par la recherche de sens ou la recherche du sens au milieu des vérités proposées ou imposées par la société. Cette recherche de sens conduit vers le déploiement de la pensée humaniste qui s'imbrique dans une communauté de recherche philosophique, porteuse de liens et garante de la loi, comme la didactique du philosopher le permet.

\section{Illustrations}

\section{Illustrations épistémologiques (objets d'enseignement et de savoirs)}

21 L'entrée en discussion philosophique avec des adolescents de lycée professionnel n'ayant pas accès à l'enseignement de la philosophie s'est effectuée sur le mode de la transmission des savoirs par le biais du groupe, à partir de thématiques contenues dans le récit. L'analyse de cet extrait porte sur la «conversion didactique » ou le « déjà-là » de la didactique clinique (Frisch, 2015). Dès la troisième séance, le parasitage de l'intentionnel et du conceptuel s'éprouve dans l'émotionnel, injecté à partir des savoirs du conte. Si la compréhension du chapitre est acquise, les interactions cognitives s'effacent avec le savoir pour laisser place à des échanges émotionnels. L'intelligence relie les adolescents, et baisse les boucliers (Richaud, 2014). L'enseignante questionne afin que lui advienne le sens de son action dans l'écriture du support destiné à susciter le désir de philosopher.

\section{Extrait corpus $\mathrm{n}^{\circ} 3$}

189: Enseignante : alors (...) la séance d'avant était mieux quand même (...) cette séance vous ne la comprenez pas \# non

190 : Dylan : ouais

191: Enseignante : vous ne comprenez pas

192: Dylan : ouais un peu

194: Dylan : celle d'avant ça allait \#, mais celle là XX

195: Enseignante : pourquoi est-ce que celle là ça va pas (...) qu'est-ce qui vous pose problème

196 : Marc : c'est trop triste

197: Dylan : ho l'autre \{approx\}

198: Enseignante : et pourquoi ça te pose problème le fait que ce soit triste

200: Enseignante : ça te pose un problème le fait qu'un conte soit triste \# Marc \#

201: Louis : ben moi \# je [ne] lis pas énormément \# alors \# heu

202: Enseignante : oui, mais spontanément tu me dis que c'est triste

203: Dylan : oui c'est triste

204: Louis : ben \# ouais \# pour les personnes qui le lisent \# ouais

205: Dylan : pour \# heu//

206: Louis : pour les personnes qui le lisent

La stratégie didactique est distillée au cours des chapitres du récit. Les dix-huit séances de discussions prévues se concentrent autour des thématiques du lien, du sens et de la loi (avec pour indicateur inversé, la honte) (Henrion-Latché, 2016). La didactique ici « 
intentionnelle» que porte l'enseignante est celle de «la gestion des interactions dites sociocognitives» (Luxembourger, Saint-Dizier de Almeida \& Specogna, 2015). Elle s'effectue à partir de concepts ou phénomènes issus des concepts de l'activité disciplinaire, ici les savoirs des métiers du bâtiment (Agostini \& Ginestié, 2012). Dans cet extrait, la métaphore du phénomène de la capillarité est mise en jeu par le procédé de métaphorisation. La compréhension de la lecture du chapitre par un élève est préalablement testée, puis le phénomène de la capillarité est questionné dans sa transposition à l'émotion humaine à partir des pierres et des végétaux.

\section{Extrait corpus $n^{\circ} 8$}

19: Enseignante : l'humidité dans le mur remonte \# et avec heu \# avec tout ce qu'i [l] se passe dans l'atmosphère \# ça donne un champignon \# en gros \# Cocha \# il se dit que \# $\mathrm{i}$ [1] veut savoir ce qu'est la capillarité \# donc la capillarité c'est un moment donné un système de pression \# ce qui se passe dans les murs \# par des canaux capillaires \# qui sont très petits ça comprime et ça fait ressortir l'eau (...) et qu'est ce qui se passe chez Cocha à ce moment-là \# quand il comprend (...)

21: Jean : à sa mère

22: Louis : à quand il était petit

24: Enseignante : ouais (...) il repense à ce qui lui est arrivé quand il était petit

25: Alan : ouais dans son village

26: Enseignante : et qu'est ce qui sort de ses yeux

27: Alan : le séisme

28: Jean : des larmes

29: Louis : des larmes

30: Enseignante : le séisme et les//

31: Jean : les larmes

32: Alan : larmes

33: Enseignante : et les larmes

34: Dylan : du liquide

L'écologie, le développement durable et la conscience adolescente sont autant de thématiques dont peuvent se saisir les élèves. Ils choisissent d'apprendre de nouveaux savoirs et de mettre en sens, en se décalant progressivement au fil des séances de l'émotionnel.

\section{Extrait corpus $n^{\circ} 8$}

64: Enseignante : alors là c'est vrai qu'[e] le \# heu \# ce qui est intéressant c'est de se dire qu'à partir d'une expérience du bâtiment \# une expérience toute bête hein \# Cocha il fait ce qu'on appelle une analogie donc il se dit voilà \# ce qui arrive au mur ça peut arriver aussi aux hommes et en plus il se rappelle \# que c'est arrivé à ses arbres \# les saules pleureurs\# les saules pleureurs ils boivent de l'eau \# et l'été par leurs feuilles \# l'eau elle recoule vous saviez ça

65: Marc: $\mathrm{mm}$

66: Louis : $\mathrm{mm}$

67: $\{$ rires\}

68: Marc : si je sais j'en ai un chez moi

69: Dylan : sûrement ouais (...) j [e ne] savais même pas que ça existait 
70: Marc : quoi les saules pleureurs

71: Dylan : ouais je [ne] sais pas c'est quoi

La didactique clinique consiste dans l'analyse de l'activité enseignante, et dans ses choix subjectifs de transmission de savoirs. Le second extrait traite d'une transposition didactique à partir du dessin technique qui consiste à tracer des plans et positionner des ouvertures et des fermetures. En partant de la notion de frontières, les adolescents élargissent leur réflexion au monde qui les entoure. Le symbolisme ouvre au réel, introduisant une réflexion collective autour d'une clinique de la mondialité s'invitant à l'école. Avant la complexité, ils n'y avaient jamais pensé.

\section{Extrait corpus $\mathrm{n}^{\circ} 7$}

96: Léa: j'ai pas compris madame quand vous disez \{sic\} que c'est quoi mettre des frontières

97: Enseignante : ben si tu veux \# tu vois par exemple entre la France et la Belgique \# c'est deux pays différents \# chut arrête arrête \# ça fait du bruit en plus en dernier \# un moment donné on met des barrières \# comme ça on se dit si les gens s'entendent pas [il] y a des barrières qui les séparent \# et puis avec l'Europe par exemple on a fait sauter ces barrières là \# donc on peut circuler librement sur tout le territoire \# donc obligatoirement [il] faut que les gens s'entendent \# tu vois dans la symbolique mettre des frontières \# et puis les retirer ou les ouvrir \# [il] y a des conséquences \# la conséquence notamment c'est comment je vais \# ben m'ouvrir à l'autre \# qui est différent (...) vous comprenez (...) vous aviez déjà réfléchi à ces problèmes de frontières 98: Léa : non

99: Enseignante : non (...) vous aviez jamais vu le monde comme ça \# ne serait-ce que le territoire européen \# non

101: Enseignante : maintenant est ce qu'on peut circuler librement en Europe

102: Louis : ouais

103: Enseignante : et qu'est-ce que ça a comme conséquences

104: Marc : tous les XX viennent dans le pays

107: Marc : tout le monde est libre dans le pays

109: Louis : XX des terroristes tout

110: Jean : faut des papiers

\section{Illustrations angle de la didactique clinique (praxéologique et psychologique)}

L'analyse de l'activité enseignante avec des adolescents empêchés de penser s'ancre dans la notion du "sujet supposé savoir ", autrement nommée "la contingence " (Frisch, 2015). La position symbolique de l'enseignant en DVP est celle qui garantit la loi. Cependant, nous assistons à des renversements de position entre animateur et adolescents, dès la première séance. Entre le prévu et l'incident, les adolescents s'improvisent support de l'activité du philosopher et renverse la didactique initialement prévue. Ils se décrètent ressource et garants des séances, et s'ouvrent aux apprentissages. 


\section{Extrait corpus $n^{\circ} 1$} 99: Enseignante : alors on va essayer de rester concentrés parce que même moi je commence un peu à m'y perdre \# qui ici// 100: Louis : je suis là madame

La didactique clinique forme et prépare l'enseignant à l'inattendu. Elle permet d'aborder la complexité telle qu'elle se présente dans les classes. Au cours de la première séance, l'analyse des relations entre l'enseignant et les adolescents est interrogée au prisme des contenus du récit. Par analogie, il est question de consulter le point de vue des élèves sur les procédés qui ont conduit l'enseignant à proposer des séances de discussions philosophiques avec des élèves au comportement complexe. Il est question d'aborder "la dialogique ordre/désordre/organisation" de la pensée complexe (Morin, 2005, p.137) par la parole des adolescents comme facteur de médiation et d'adaptation. La visée est résiliente.

\section{Extrait corpus $n^{\circ} 1$}

307: Enseignante : alors moi je me souviens \# quand je suis arrivée dans cette classe en début d'année \# j'ai vu des élèves (...) des élèves pas très gentils \# qui excluaient un de leur camarade \# vous vous souvenez (...) pourquoi moi j [e n'] ai pas réagi comme tout le monde

308: Louis : vous n'avez pas tenu compte du bonheur

309: Enseignante : non ce n'est pas du bonheur

310: Dylan : vous n'avez pas jugé sans connaître

$33 \mathrm{Au}$ terme de la troisième séance, l'analyseur de «l'impossible à supporter» de la didactique clinique, qui consiste à se rapporter aux expériences délétères ou traumatisantes enseignantes, se renverse à nouveau. D'une classe désorganisée, les adolescents ont adhéré au protocole de discussions et ont adopté la posture d'écoute attentive attendue dans les DVP. L'attendu de la complexité des séances d'enseignement se mue en attente des consignes enseignantes. Contre toute attente, les élèves sont devenus attentifs et demandent pour prendre la parole. Les bénéfices des séances partagées "secouent la paresse d'esprit, c'est une leçon que donne la pensée complexe » (Morin 2005, p.110)

\section{Extrait corpus $n^{\circ} 3$}

4 59: Enseignante : pourquoi alors (...) moi j'ai besoin d'entendre des arguments (...) alors on va discuter autour du conte \# Cocha il savait que \# heu \# il s'est construit une personnalité en fait \# en se disant que s'il est le fils d'un guerrier \# qu'il est brave \# il va pouvoir faire plein de choses (...) on se rend compte qu'il n'a pas pu sauver personne (...) donc selon vous 60: \{Silence\}

61: Enseignante : [il] y'a rien//

62: Dylan : on vous écoute madame

63: Enseignante : mais c'est à moi de vous écouter normalement

5 Les modalités du penser des échanges discursifs en DVP, analysés à l'aide des descripteurs de la didactique clinique, s'inscrivent dans l'esprit critique. S'engager en clinique lors de séances de discussion avec des adolescents dysfonctionnels, c'est aussi 
concevoir la libération d'une parole scolaire préalablement confisquée et des prises de conscience incitant à la reprise interlocutoire de l'enseignant. Ces rebonds doivent s'orienter vers une dialectique des pensées et des comportements, puisque «les prises de conscience nécessitent l'autocritique, mais celle-ci a besoin d'être stimulée par la critique » (Morin, 2005, p.149).

\section{Extrait corpus $n^{\circ} 13$}

\section{le bonheur}

333: Dylan : $\mathrm{j}[\mathrm{e}$ ne] sais pas

334: Alan :j'en sais rien \# parce qu'ils [ne] sont pas comme nous

335: Enseignante : parce qu'ils ne sont pas comme vous

336: Dylan : ouais plus on tombe \# plus on s'endurcit

\section{Conclusion}

La didactique clinique, pratique émergente, offre des pistes intéressantes dans l'analyse de l'activité enseignante et dans le rapport enseignant/enseignés, au sein de classes complexes ou difficiles, en faveur des adolescents en situation d'apprentissage de la discussion philosophique. Ces séances permettent à un enseignement complet de se mettre en œuvre, impliquant des savoirs disciplinaires autour d'une intelligence collective régulant les interactions par l'apprentissage du bien pensé. Par l'analyse des corpus produits, il est possible de repérer de nouveaux indicateurs discursifs ou conversationnels définissant les questionnements fonctionnels des participants, mais également structurels. S'interroger sur soi, sur les autres, en groupe, autour de savoirs conceptualisés, de notions clés, de faits ou d'opinions offre aux adolescents des espaces de récupération psychique, de liberté de penser, loin des parasitages dogmatiques ou des émotions. Par l'activité discursive et la reprise interlocutoire de l'enseignant, l'oral se développe, le langage s'affine et l'activité partagée enseignant/adolescent régule les rapports d'une communauté en activité de recherche philosophique. Loin de la complexité qui exprimait "notre embarras, notre confusion, notre incapacité de définir de façon simple, de nommer de façon claire, de mettre de l'ordre dans nos idées " (Morin, 2005, p. 9), ces séances mettent en œuvre des interactions sociocognitives progressives, conduisant vers les attendus de fin de formation scolaire et professionnelle lors du passage à l'oral. En indiquant ce qu'il importe d'identifier, en incorporant de nouvelles méthodes de pensée, en proposant des opérations mentales à effectuer pour penser en dialectique, le devoir d'éducation, du côté de l'enseignant comme de celui des élèves, devient celui «d'armer chacun dans le combat vital pour la lucidité» (Morin, 1999, p.37). L'enseignant, sans devenir son propre objet d'analyse, effectue ses choix et affine sa pratique par les retours d'expérience de séances de discussions. La didactique clinique constitue un cadre théorique de référence doté d'outils efficients pour analyser les nouvelles pratiques émergentes nécessaires dans les métiers de l'éducation et de l'humain en faveur de la scolarité. Dans une société complexe, en mutation, privilégier la réflexion, l'intelligence et l'articulation des savoirs semble à la fois vecteur d'intelligence et facteur de progrès. C'est une nouvelle humanité qui advient. "Cette nouvelle humanité qui est en train de naitre doit être une humanité de débat. Cela est très fatigant, mais très passionnant, c'est la source de la vie » (Cyrulnik \& Morin, 2010, p. 45). 


\section{BIBLIOGRAPHIE}

Agostini M. \& Ginestié J. (2012). Réflexion sur l'utilité et le sens des enseignements technologiques. Penser l'éducation, 32, 15-29.

Alexandre, L. (2017). La guerre des intelligences comment l'intelligence artificielle va révolutionner l'éducation. Paris : J.C Lattès.

Astolfi, J.P. \& Delevay, M. (1989). La didactique des sciences. Paris : Presses Universitaires de France.

Auriac-Slusarczyk, E. (2010). Les interactions à l'école où en sommes-nous ? Psychologie de l'Interaction, 27-28.

Auriac-Slusarczyk, E. \& Henrion-Latché, J. (2018). Résilience et philosophie : du courage en éducation. Paris : L'Harmattan.

Blanquer, J-M. \& Morin, E. (2020). Quelle école voulons-nous. La passion du savoir. Paris : Odile Jacob, éditions Sciences Humaines.

Boimare, S. (2008). Ces enfants empêchés de penser. Paris : Dunod.

Catheline, N. \& Marcelli, D. (2011). Ces adolescents qui évitent de penser. Pour une théorie du soin avec médiation. Paris : Erès.

Coutel, C. (2000). L'école de la philosophie. Nantes : éditions pleins feux.

Cyrulnik, B. (2004/2007). Parler d'amour au bord du gouffre. Paris, France : Odile Jacob.

Cyrulnik, B. \& Morin, E. (2010). Dialogue sur la nature humaine. Paris : éditions de l'Aube.

Daniel, M.F. (1997). La philosophie et les enfants. Paris-Bruxelles : De Boeck Université

Daniel, M.F. (2012). Que signifie philosopher ? La complexité épistémologique d'une pensée critique dialogique : un critère du philosopher, in Philosophèmes

Daniel, M-F. (2007). L'apprentissage du philosopher et le processus développemental d'une pensée critique dialogique. In M. Tozzi (dir.). Apprendre à philosopher par la discussion. Pourquoi ? Comment? (p.123-137). Bruxelles : Éditions De Boeck Université.

Daniel, M-F. \& Delsol, A. (2010). Apprendre à dialoguer de manière critique en maternelle. Une étude de cas québécoise. Psychologie de l'interaction, numéro spécial: Les interactions à l'école: Où en sommes-nous?,p.64-97.

Daniel, M-F. \& Gagnon, M. (2011). A developmental model of dialogical critical thinking in groups of pupils aged 4 to 12 years. Creative Education, 2 (5), p.418-428.

Dehaene, S. (2018). Apprendre! Les talents du cerveau au défi des machines. Paris : Odile Jacob.

Derivois, D. (2010). Les adolescents victimes/délinquants. Observer, écouter, comprendre, accompagner. Bruxelles : De Boeck.

Frisch, M. (Dir.) (2018). Construction de savoirs et de dispositifs. Paris : L'Harmattan.

Frisch, M. (2016). Émergences en didactiques pour les métiers de l'humain. Paris : L'Harmattan.

Frisch, M. \& Paragot, J-M. (2015). Qu'est-ce que la didactique clinique ? Garelli Déborah. En ligne : https://wikidocs.univ-lorraine.fr/pages/viewpage.action?pageId=117999489 
Frisch, M. (2008). Didactique des disciplines et didactique professionnelle - Intervention au séminaire du GRCDI. (Groupe de Recherche sur la Culture et la Didactique Informationnelles).

Frisch, M. (2007). Disciplinarisation et didactisation. Esquisse, p.155-165.

Gabriel, M. (2019). Pourquoi la pensée humaine est inégalable. Paris : édition J.C. Lattès.

Go, N. (2007). Analyse didactique d'une discussion à visée philosophique en CM1 : l'action du professeur. In Tozzi, M. La discussion en éducation et en formation. Un nouveau champ de recherche, $\mathrm{p}$. 23-35. Paris : L'Harmattan.

Henrion, J. (2015). Résilience et discussion à visée philosophique en lycée professionnel : en quoi la DVP s'inscrit comme médiation facilitant un processus de résilience à l'école. Les Cahiers du CERFEE.

Henrion, J. (2012). Les aventures de Cocha l'indigène au pays du Génie Civil. Non publié, http:// philosophemes.univ-bpclermont.fr/2016da9ca964bd31d2e879ecea54ff00/cocha-et-b\%C3\%A9niau-pays-du-g\%C3\%A9nie-civil, 47 pages.

Henrion-Latché, J. (2019). Esprit critique en lycée professionnel : l'exemple des discussions à visée philosophique comme pratiques dialogiques de résilience culturelle. Grenoble : revue en ligne, site de L'OUVROIR LITT\&ARTS.

Henrion-Latché, J. (2016). Résilience culturelle et discussion à visée philosophique : étude suivie de sept élèves en lycée professionnel. Thèse de doctorat. Sciences de l'éducation. Université Clermont Auvergne.

Henrion-Latché J. \& Auriac-Slusarczyk E. (2020a). Étude sémantico-pragmatique du discours au profit d'un décryptage du parcours psychologique suivi d'adolescents fragilisés. Proposition d'une démarche de codage de discours. Revue Corpus, en ligne.

Henrion-Latché, J. \& Auriac-Slusarczyk, E. (2020b). Philosophie et résilience testées auprès d'adolescents dysfonctionnels. In Metz, C. (dir.). L'Enfant non conforme. Strasbourg : Presses Universitaires de Strasbourg. Collection famille, psyché, sociétés.

Henrion-Latché, J., Auriac-Slusarczyk, E. \& Tozzi, M. (2019). Exercer une fraternité au lycée professionnel : expérience de philosophie à partir d'un conte ad hoc. Revue Penser l'éducation, $\mathrm{n}^{\circ}$ 42, p.53-67.

Henrion-Latché, J. \& Frisch, M. (2019). Professionnalisation des apprenants : prise en compte de l'influence de la variation sexolectale dans les métiers du bâtiment et la formation professionnelle. Revue Éducation permanente.

Henrion, J. \& Tozzi, M. (2016). Discussion à visée philosophique et résilience : déontologie, pratique de l'enseignant et style d'animation dans la conduite de discussions philosophiques à visée résiliente. Revue des sciences de l'éducation, vol. 42, n³, 2016, p.168-186. URI : http:// id.erudit.org/iderudit/1040088ar

Jellab, A. \& Marsollier, C. (2018) Bienveillance et bien-être à l'école : pour une école humaine et exigeante. Boulogne-Billancourt : Levrault-Berger.

Johsua, S. \& Dupin, J.J. (1993). Introduction à la didactique des sciences et des mathématiques. Paris : Presses Universitaires de France.

Lecomte, J. (2010). Guérir de son enfance. Paris, France : Odile Jacob.

Lévine, J. (2008). L'enfant philosophe, avenir de l'humanité ? Issy-les-Moulineaux : ESF.

Levine, J. \& Moll, J. (2004). JE est un autre. Pour un dialogue pédagogie-psychanalyse. Lyon : ESF. 
Lipman, M. (1991/1995). Thinking in Education, traduction, N. Decostre, À l'école de la pensée : Enseigner une pensée holistique. Louvain-la-Neuve : De Boeck Université.

Lipman, M., Sharp, A-M., \& Oscanyan, F. S. (1980). Philosophy in the classroom. Philadelphia PA: Temple University Press.

Lipman, M. (1978). L'enfant qui vient de l'avenir. La découverte d'Harry Stottlemeier. Paris : Vrin.

Luxembourger, C., Saint-Dizier -De Almeida, V. \& Specogna, A. (2015). La discussion philosophique comme modèle pour former les enseignants à la gestion des interactions sociocognitives. In Auriac-Slusarczyk, E. \& Coletta, J.M. Les ateliers de philosophie : une pensée collective en acte. (p. 360-374). Clermont-Ferrand : Presses Universitaires Blaise Pascal.

Morin, E. (1999). Les sept savoirs nécessaires à l'éducation du futur. Paris : Seuil.

Morin, E. (1990/2005). Introduction à la pensée complexe. Paris : Seuil.

Morin, E. (2015). Penser global, L'homme et son univers. Paris : Robert Laffont.

Moro, M.R. (2017). Et si nous aimions nos ados ? Paris, France : Bayard.

Richaud, R-L. (2014). Psychanalyse maïeutique à l'adolescence : Sortir de la non-pensée. Auxerre : $\mathrm{H}$ Diffusion.

Sasseville, M. (2019). Pensée critique, créatrice et attentive. La pratique de la pensée complexe en communauté de recherche philosophique. https://philoenfant.org/2019/01/15/pensee-critiquecreatrice-et-attentive/

Sasseville, M. \& Gagnon, M. (2007). Penser Ensemble à l'école : Des Outils Pour l'observation D'une Communauté de Recherche Philosophique en Action. Presses Université Laval.

Taddei, F. (2018). Apprendre au XXIe siècle. Paris : Calmann-Lévy.

Troadec, B. (2007). Psychologie culturelle. Le développement cognitif est-il culturel ? Paris : Belin Sup

Tozzi, M. (2011). Animer une discussion à visée philosophique en classe. L'apprentissage $d u$ philosopher, http://www.philotozzi.com/2011/03/439/

Tozzi, M. (2009). La didactique de la philosophie en France - 20 ans de recherche (1989-2009) », Les Cahiers du Cerfee n² 26, Montpellier 3, 2009.Diotime n 39, janv. 2009.

Tozzi, M. (2007). Apprendre à philosopher par la discussion : Pourquoi ? Comment ? Louvain-la-Neuve, Belgique : De Boeck supérieur.

Tozzi, M. et Étienne, R. (2004). La discussion en éducation et en formation : un nouveau champ de recherches (Vol. 1-1). Paris : l'Harmattan.

Tozzi, M. \& Solère-Queval, S. (2004). Le rôle du maître dans la discussion à visée philosophique à l'école primaire et au collège. In Tozzi, M. (Dir.), La discussion en éducation et en formation. Un nouveau champ de recherche. Paris : L'Harmattan.

Tozzi, M., Meirieu, P. \& Huguet, F. (2002). Penser par soi-même : initiation à la philosophie. Lyon : Chronique sociale.

Trognon, A. (1995). La fonction des actes de langage dans l'interaction : l'exemple de l'intercompréhension en conversation. Lidl, 12, pp.67- 85.

Trognon, A. (1991). La structuration interlocutoire des groupes. Revues de psychothérapie psychanalytique des groupes, 17, 78-92. 
Vavassori, D. \& Harrati, S. (2018). La psychologie clinique au défi des symptômes contemporains. Paris : In press éditions.

\section{NOTES}

1. Ces corpus sont disponibles sur la plateforme collaborative Philosophèmes: https:// philosophemes.msh.uca.fr/accueil

2. https://wikidocs.univ-lorraine.fr/display/IUFMIDEKI/Accueil

3. Désormais IA

\section{RÉSUMÉS}

A l'école et particulièrement dans certains lycées dits sensibles, les problématiques de violence, des marqueurs de délinquance affichent autant de refus de savoir et de penser que de marqueurs de vulnérabilités d'adolescents en lien avec la complexification de la société (Jellab \& Marsollier, 2018). Si la résilience est visée par le rebond de la pensée, celle-ci peut être portée par des séances porteuses couvertes par une didactique clinique inscrite dans les discussions philosophiques. Cet article illustre comment savoir, intelligence et complexité s'articulent dans la mise en œuvre d'une didactique clinique au service du philosopher avec des adolescents empêchés de penser.

In so-called sensitive high schools, issues of violence, markers of delinquency display as much refusal to know and think as markers of adolescents vulnerabilities related to complexity of society (Jellab \& Marsollier, 2018). If resilience is targeted by the rebound of thought, it can be carried by carrier sessions covered by a clinical didactic written in philosophical discussions. This article illustrates how knowledge, intelligence and complexity are articulated in the implementation of a clinical didactics at the service of the philosopher with adolescents prevented from thinking.

\section{INDEX}

Mots-clés : intelligence, discussion philosophique, adolescents, didactique, didactique clinique, résilience

Keywords : intelligence, philosophical discussions, adolescents, didactics, clinical didactics, resiliency

\section{AUTEUR}

\section{JOHANNA HENRION-LATCHÉ}

Université de Reims Champagne-Ardenne (URCA), composante INSPE, laboratoire CEREP, EA 4692 